\title{
Post-Processing a Conceptual Rainfall-Runoff Model with an LSTM
}

\author{
Grey S. Nearing ${ }^{1,2}$, Alden Keefe Sampson ${ }^{1}$, Frederik Kratzert ${ }^{3}$, Jonathan M. \\ Frame $^{2}$ \\ ${ }^{1}$ Upstream Tech, Natel Energy Inc.; Alameda, CA USA \\ 2 Department of Geological Sciences, University of Alabama; Tuscaloosa, AL USA \\ ${ }^{3}$ LIT AI Lab \& Institute for Machine Learning, Johannes Kepler University; Linz, Austria
}

\begin{abstract}
Machine learning is becoming an increasingly important part of streamflow forecasting, but as these models to date lack a physical basis, there is a potential that they may produce values that are not realistic. We tested a simple post-processing strategy that uses the outputs from a calibrated conceptual model (the Sacramento Soil Moisture Accounting Model with Snow-17; SAC-SMA) as inputs into a a Long Short Term Memory Network (LSTM). Overall, the SAC-SMA model was improved substantially, while post-processing offered only minor improvements relative to the standalone LSTM. SAC-SMA performance was improved in catchments with more snow. The standalone LSTM was improved in terms of long-term bias, which is likely because the LSTM is not constrained by conservation principles.
\end{abstract}

\section{Introduction}

As the volume and accessibility of large hydrological data sets increases, deep learning is becoming an increasingly important technology for simulating rainfall-runoff processes and estimating streamflow. Recent research has shown that Long Short Term Memory networks (LSTMs; Hochreiter \& Schmidhuber, 1997) provide streamflow predictions with accuracy that rivals or exceeds traditional hydrology models (Kratzert, Klotz, Herrnegger, et al., 2019; Kratzert, Klotz, Shalev, et al., 2019), however all types of machine learning, including LSTMs, lack an explicit physical basis.

The hydrological sciences community is currently exploring several strategies for combining physical models and physical understanding with machine learning (e.g., Daw et al., 2020; Nearing et al., 2020; Pelissier, Frame, \& Nearing, 2020), however one of the simplest methods is post-processing. Post-processing is where outputs from a conceptual or physics-based model are used as inputs to the machine learning model. Post-processing is at least potentially useful for improving prediction accuracy in cases where the conceptual model provides information that the machine learning model was not able to extract from data. More importantly, we can use various types of diagnostic tools to help understand where and when the conceptual model provides extra information, and use this as a guide for understanding the strengths and deficiencies of different types of models.

Corresponding author: Grey Nearing, grey@upstream.tech 
In this paper, we investigated post-processing the Sacramento Soil Moisture Accounting model (SAC-SMA; Burnash, Ferral, \& McGuire, 1973) with an LSTM. SACSMA is a calibrated lumped conceptual model that forms the basis of the US National Weather Service Community Hydrologic Prediction System (Roe et al., 2010), and was one of the models that was benchmarked by Kratzert, Klotz, Herrnegger, et al. (2019).

\section{Methods}

\subsection{Data \& Models}

Data used for this study are from the Catchment Attributes and Meteorology for Large-Sample Studies data set (CAMELS; Addor, Newman, Mizukami, \& Clark, 2017; Newman et al., 2015) curated by the US National Center for for Atmospheric Research. All data are at a daily timestep for the period 1980-2008 over 671 basins in the continental United States. We used 531 of the 671 basins - these were the same basins used by Newman et al. (2015), who excluded basins with large discrepancies in different methods for measuring basin area, and also excluded basins that were larger than $2,000 \mathrm{~km}^{2}$.

Our LSTMs take two types of inputs: daily meteorological forcings and static catchment attributes. Meteorological forcings include daily maximum and minimum temperatures, daily cumulative precipitation, solar radiation, and vapor pressure deficit. CAMELS includes three meteorological data sets: NLDAS, Daymet, and Maurer. following Kratzert, Klotz, Hochreiter, and Nearing (2020), we used all three as inputs into the LSTM, for a total of fifteen daily forcings. We used twenty-seven catchment attributes from the CAMELS dataset related to climate, vegetation, topography, geology, and soils. These are described in more detail by Addor et al. (2017). Catchment attributes are static for each basin (do not change in time), and were appended to the meteorological forcings at each timestep as inputs into the LSTM (Kratzert, Klotz, Shalev, et al., 2019).

The LSTM as a hydrological model was described in detail by Kratzert, Herrnegger, Klotz, Hochreiter, and Klambauer (2019). It is a type of recurrent neural network with an explicit state space. Kratzert, Klotz, Shalev, et al. (2019) demonstrated that the gates of the LSTM can be trained to learn catchment similarity, and Kratzert, Herrnegger, et al. (2019) demonstrated that the states of a trained LSTM track unobserved hydrologically relevant variables. We trained an ensemble of $N=10$ LSTMs to account for randomness in the weight initialization. The training period was October 1, 1999 through September 30, 2008 (9 years) the test period was October 1, 1989 through September 30 (9 years), 1998. This is similar to the training and test periods used by Newman et al. (2015) and Kratzert, Klotz, Herrnegger, et al. (2019) except that we used one less year of data for the test period in order to avoid bleedover from the 365-day LSTM look-back period. LSTMs were trained and tested using sequence-to-one prediction with a 365-day lookback, and each of the $N=10$ LSTMs was trained on all training data from all 531 basins.

SAC-SMA is lumped conceptual model that must be calibrated separately for each basin (unlike the LSTM, which is trained on all data from all basins). SAC-SMA was coupled with the Snow-17 snow module and a unit hydrograph router. SAC-SMA calibrations were done by Newman et al. (2015), who used shuffled complex evolution (Duan, Gupta, \& Sorooshian, 1993) with ten random seeds, meaning that there was an ensemble of $N=10$ separate calibrations for each basin and for each of three meteorological data sets, for a total SAC-SMA ensemble of size $N=30$ for each basin.

SAC-SMA outputs used as inputs into the LSTM were: routed streamflow (QQ), snow water equivalent (SWE), combined rain and snowmelt (RAIM), potential evapotranspiration (PET), actual evapotranspiration (ET), upper zone tension water content (UZTWC), upper zone free water content (UZFWC), lower zone tension water content 
(LZTWC), lower zone free supplemental content (LZFSC), lower zone free primary content (LZFPC), and additional impervious water content (ADIMC).

\subsection{Experimental Design}

All statistics reported in the Results section were calculated over the test period using the ensemble-averaged hydrograph in each CAMELS basin. SAC-SMA statistics were calculated over hydrographs that were the the average of $N=30$ separate SACSMA calibrations in each basin, and the LSTM statistics were calculated over hydrographs that were the the average of $N=10$ separate LSTMs in each basin.

LSTMs were trained with and without the SAC-SMA outputs listed in Section 2.1. These models were benchmarked, along with the standalone SAC-SMA, using metrics related to (1) overall performance (Nash-Sutcliffe Efficiency), (2) overall bias, and (3) error in the timing of peak flow (average number of timesteps difference between simulated and observed peak flow). The models were also benchmarked using a number of hydrological signatures, as outlined by Kratzert et al. (2020). Hydrological signatures are statistics that emphasize different aspects of a hydrograph (Addor et al., 2018; McMillan, Westerberg, \& Branger, 2017). We calculated the following hydrological signatures over both simulated and observed hydrographs: high and low flow frequencies and durations (high_q_freq, high_q_dur, low_flow_freq, low_flow_dur), 5th and 95th percentile flows $(q 5, q 95)$, mean flow (q_mean), mean half flow date ( $\left.h f d \_m e a n\right)$, baseflow index, and slope of the flow duration curve $\left(\right.$ slope $\left._{-} f d c\right)$. We report the correlation (over 531 basins) between simulated and observed signatures.

In addition to benchmarking, we measured the relative contributions of different inputs into the LSTM using integrated gradients (Sundararajan, Taly, \& Yan, 2017). Integrate gradients are a type of sensitivity analysis that are relatively insensitive to low gradients (e.g., at the extremes of neural network activation functions). Integrated gradients are calculated separately for each input, at each timestep, for each lookback timestep, in each basin. This means that for 9 years of test data with a 365-day lookback there are about 1.2 million integrated gradients per input, per basin.

Finally, to help assess when and where post-processing helps, we used random forest regression to predict differences in performance scores between the standalone models (LSTM and SAC-SMA) vs. the LSTM post-processor. We report the correlation $\left(r^{2}\right)$ of out-of-sample random forest predictions of post-processing improvements using k-fold cross validation $(k=20)$ over the 531 basins. We tested predictability in this way using three sets of regressors: (1) static catchment attributes and hydrological signatures, (2) integrated gradients related to all LSTM inputs, and (3) basin-averaged SAC-SMA variables.

\section{Results}

\subsection{Benchmarking}

Global benchmarking results are shown in Figure 1. The takeaway from these figures is that the LSTM post-processor improved the performance of SAC-SMA significantly according to all three metrics, however using SAC-SMA outputs improved the LSTM only in reducing the overall bias. Not all basins were improved, however. As shown in the scatterplots in Figure 1, SAC-SMA gave better results against all three metrics in a small number of basins.

Figure 2 shows the spatial distribution of performance increases/decreases of the augmented LSTM (with SAC-SMA inputs) as compared to the standalone SAC-SMA. The LSTM improved the NSE scores in 441 of 531 basins (83\%), overall bias in 270 of 

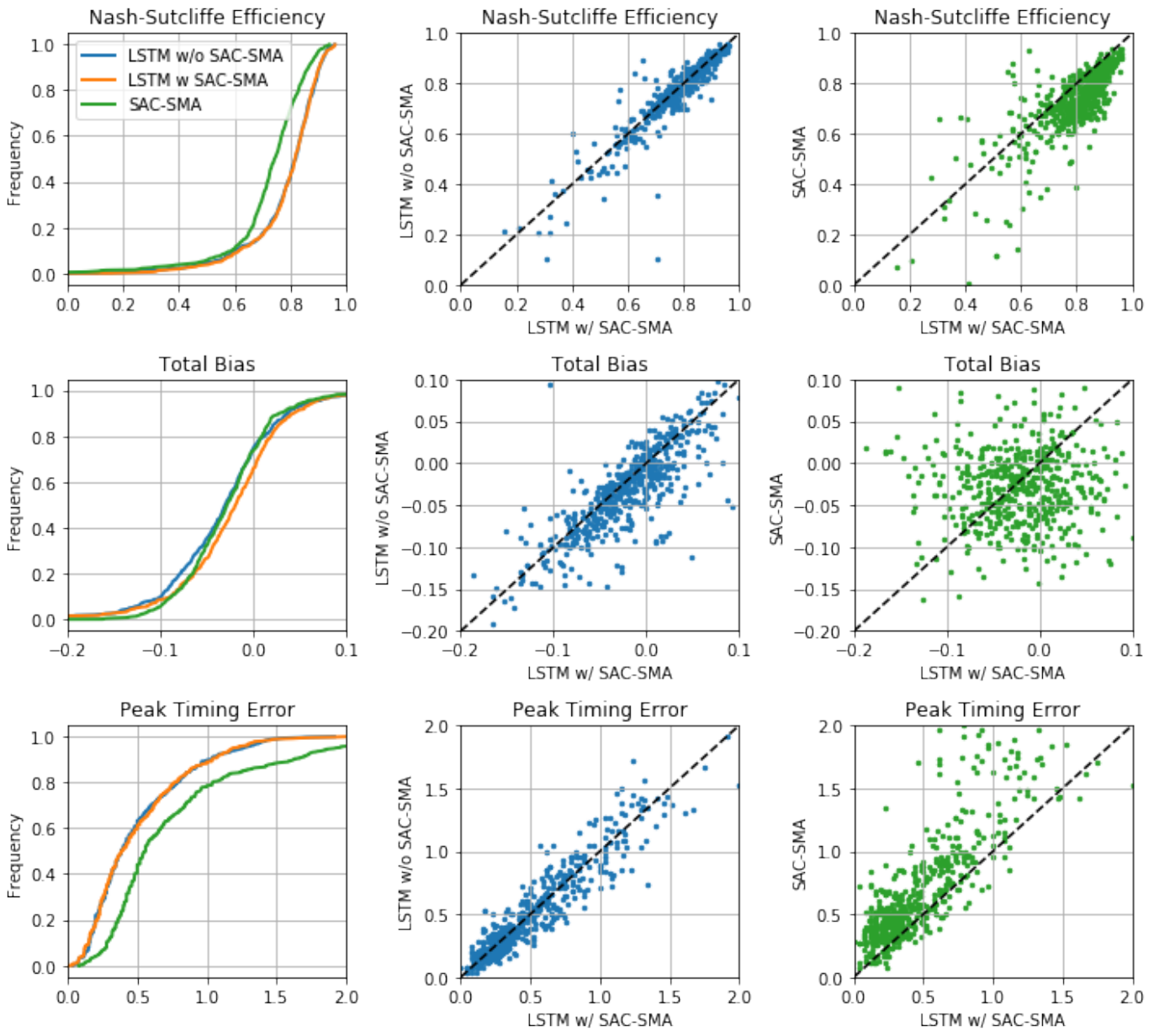

Figure 1. Cumulative distributions of three performance metrics over 531 CAMELS basins. Models with an NSE close to one are better, whereas models with bias and peak timing errors close to zero are better. The takeaway from these figures is that the LSTM post-processor improved the performance of SAC-SMA significantly according to all three metrics, however using SAC-SMA outputs improved the LSTM only in reducing the overall bias. 

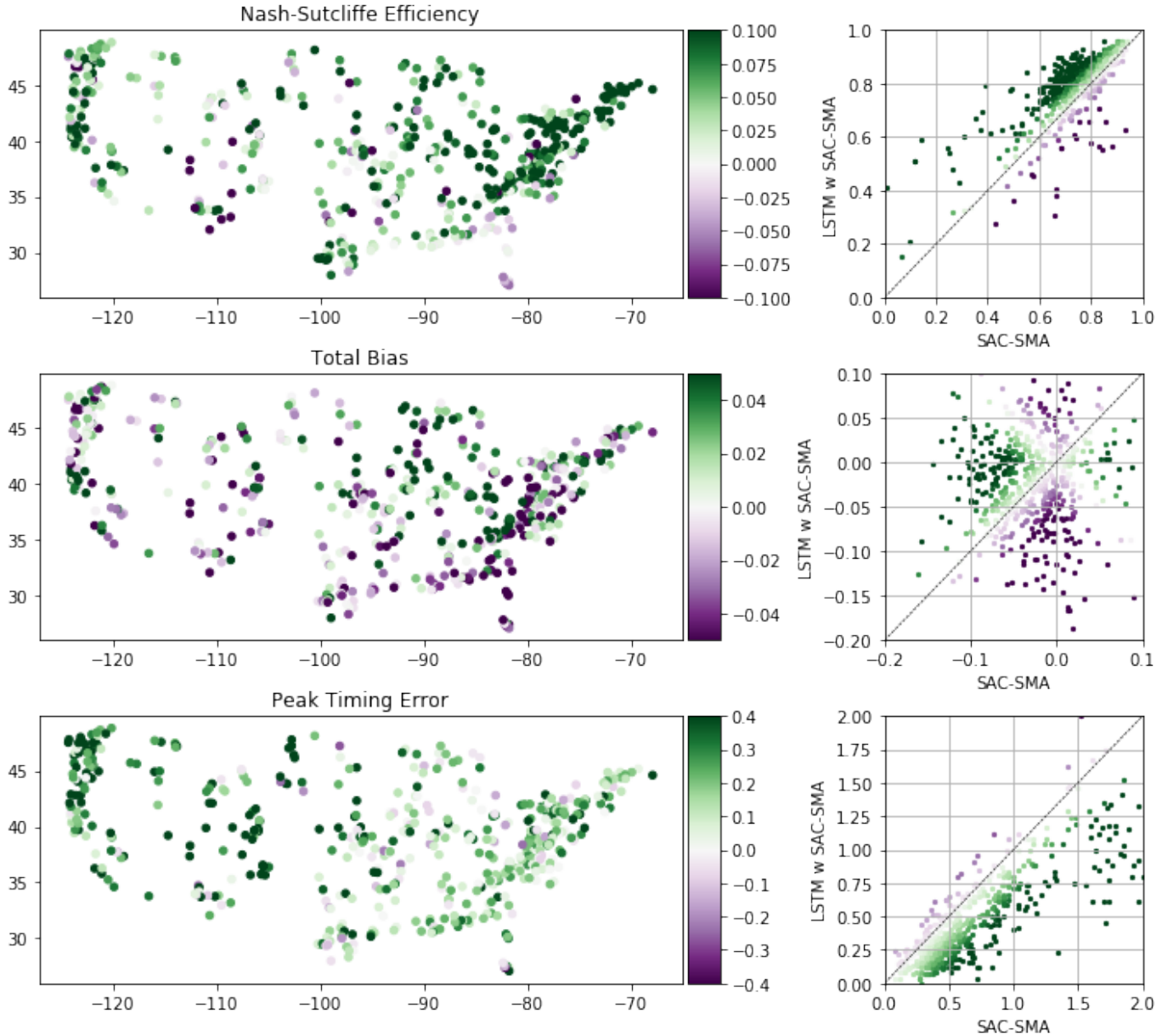

Figure 2. Spatial benchmarking plots showing basin-specific improvement/degradation due to post-processing the SAC-SMA outputs. Purple colors indicate basins where SAC-SMA performs better and green colors indicate basins where the LSTM post-processor performs better.

531 basins (51\%), and peak timing in 434 of 531 basins (82\%). On average, performance improved by $21 \%$ (NSE), degraded by $230 \%$ (bias), and improved by $29 \%$ (peak timing).

Similarly, Figure 3 shows the spatial distribution of performance increases/decreases of the augmented LSTM (with SAC-SMA inputs) as compared to the standalone LSTM (without SAC-SMA inputs). The LSTM improved the NSE score in 276 of 531 basins (52\%), overall bias in 313 of 531 basins (59\%), and peak timing in 214 of 531 basins (40\%). On average, performance degraded by $2 \%$ (NSE), improved by $101 \%$ (bias), and improved by $1 \%$ (peak timing).

The main takeaways from Figures 2 and 3 is that while the LSTM post-processor increases hydrograph correlation (NSE) and timing, SAC-SMA has a lower overall bias. This is because SAC-SMA conserves mass in the long-term. Letting the LSTM learn from SAC-SMA outputs improves the overall LSTM bias, but LSTM is still not constrained by conservation laws.

Figure 4 compares the performance of the three models in reproducing several hydrological signatures. This plot shows the correlation between the signatures of observed and simulated hydrographs. The performance of all three models is relatively similar, 

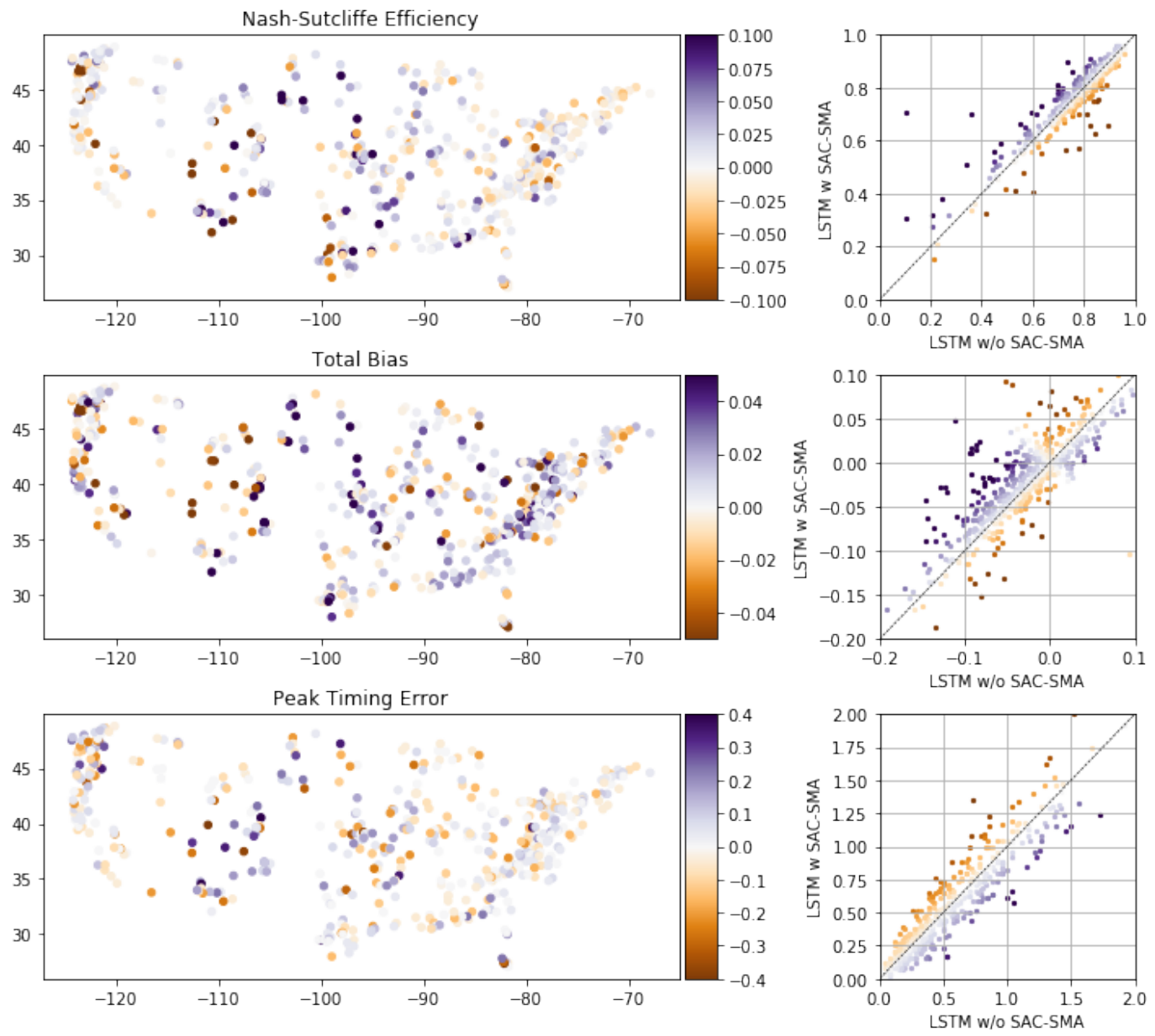

Figure 3. Spatial benchmarking plots showing basin-specific improvement/degradation to the LSTM due to using SAC-SMA outputs. Purple colors indicate basins where the standalone LSTM performs better and green colors indicate basins where the post-processing LSTM performs better. 


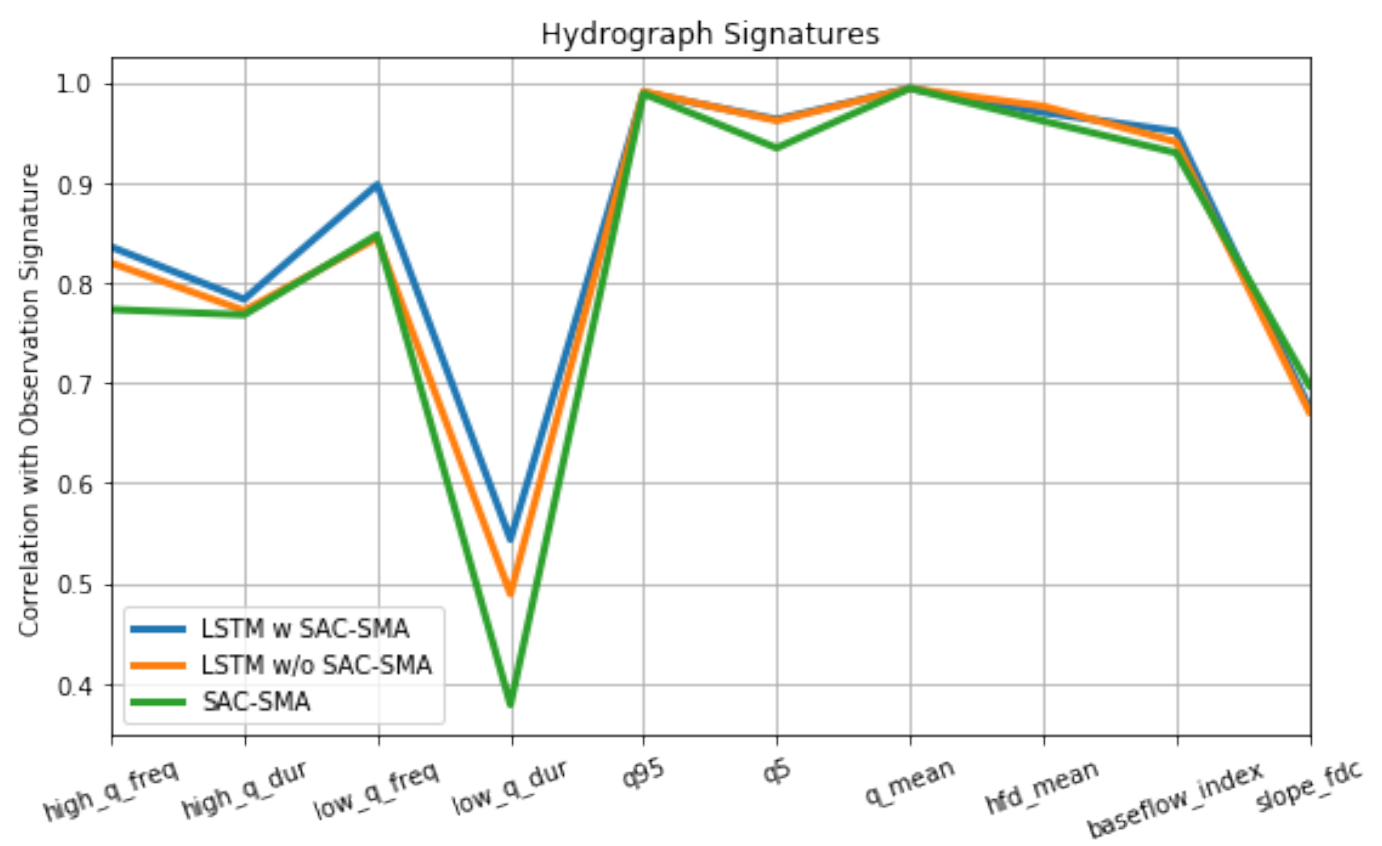

Figure 4. Correlations between simulated and observed hydrologic signatures.

except that the post-processing LSTM improved over both SAC-SMA and the standalone LSTM in predicting low-flow duration and frequency.

\subsection{Sensitivity}

Figure 5 shows the basin- and time-averaged integrated gradients as a function of the first five lookback timesteps (i.e., days before present) summed over all SAC-SMA related inputs and all meteorological forcing inputs. What is notable here is that although the SAC-SMA related inputs have a lower effect at the present timestep, in the rising limb the SAC-SMA related inputs have a larger effect than the meteorological inputs at a lag of one timestep. This is because several of the SAC-SMA outputs are actually state variables with persistence (state variables are the modeled variables that are necessary to calculate model outputs at the next timestep). Likewise, Figure 6 shows the same global averaged sensitivities for only the SAC-SMA related inputs. This figure groups categories of inputs, and here we see that it is primarily the lower zone soil moisture states that drive this persistence.

Figure 7 shows the relative contributions of each category of inputs broken out over basins. This figure shows that there is diversity between basins in terms of how the LSTM post-processor combines the different input data. Although meteorological forcings are generally more influential than SAC-SMA related inputs in most basins, the SAC-SMA variables do constitute a significant portion of the sensitivity of the LSTM model, even though the improvement scores from Section 3.1 showed relatively little change in overall performance metrics relative to an LSTM without SAC-SMA. This is because there is substantial redundancy between the SAC-SMA related variables and the meteorological forcings, due to the fact that SAC-SMA uses largely the same forcing data as the LSTMs.

Finally, Figure 8 shows the spatial distribution of the relative contributions of different categories of SAC-SMA related inputs. It's difficult to see any spatial patterns vi- 

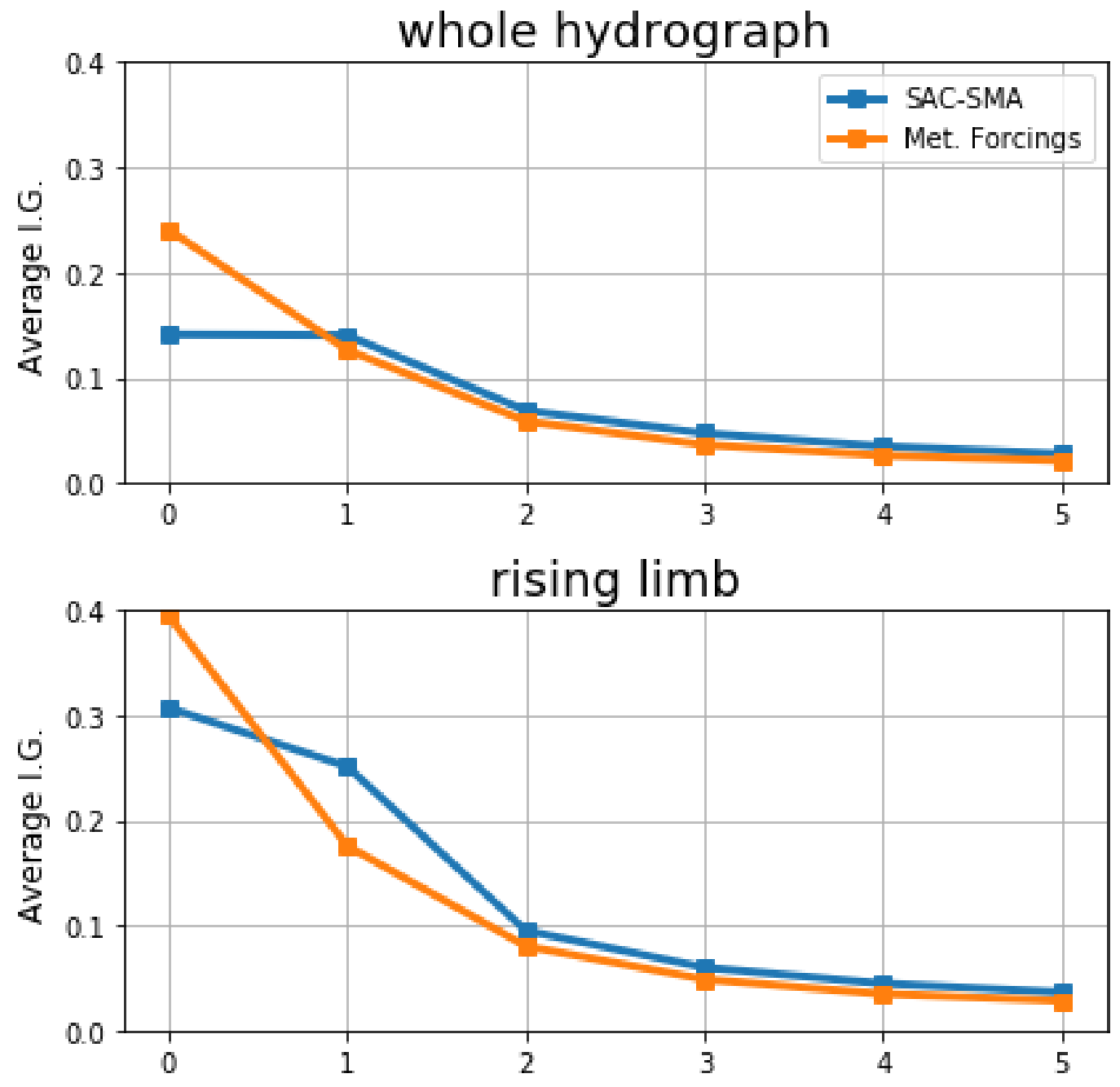

falling limb

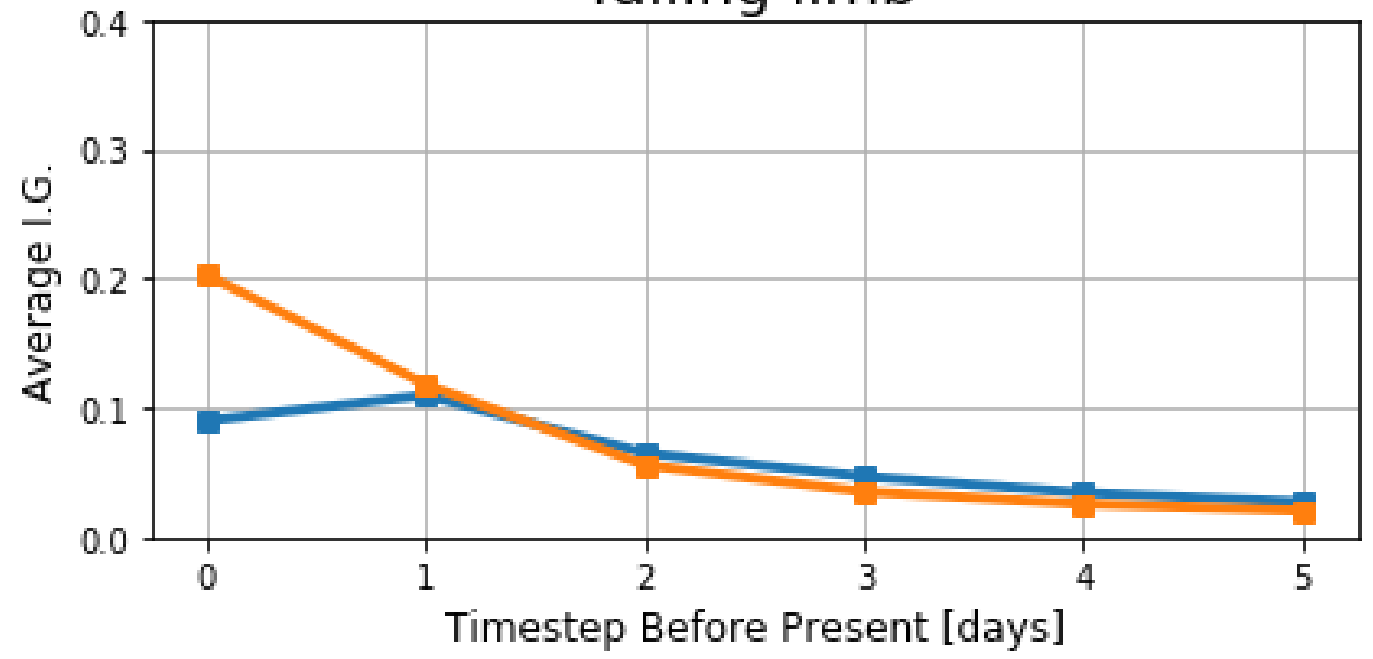

Figure 5. Basin- and time-averaged integrated gradients as a function of the first five lookback timesteps. 

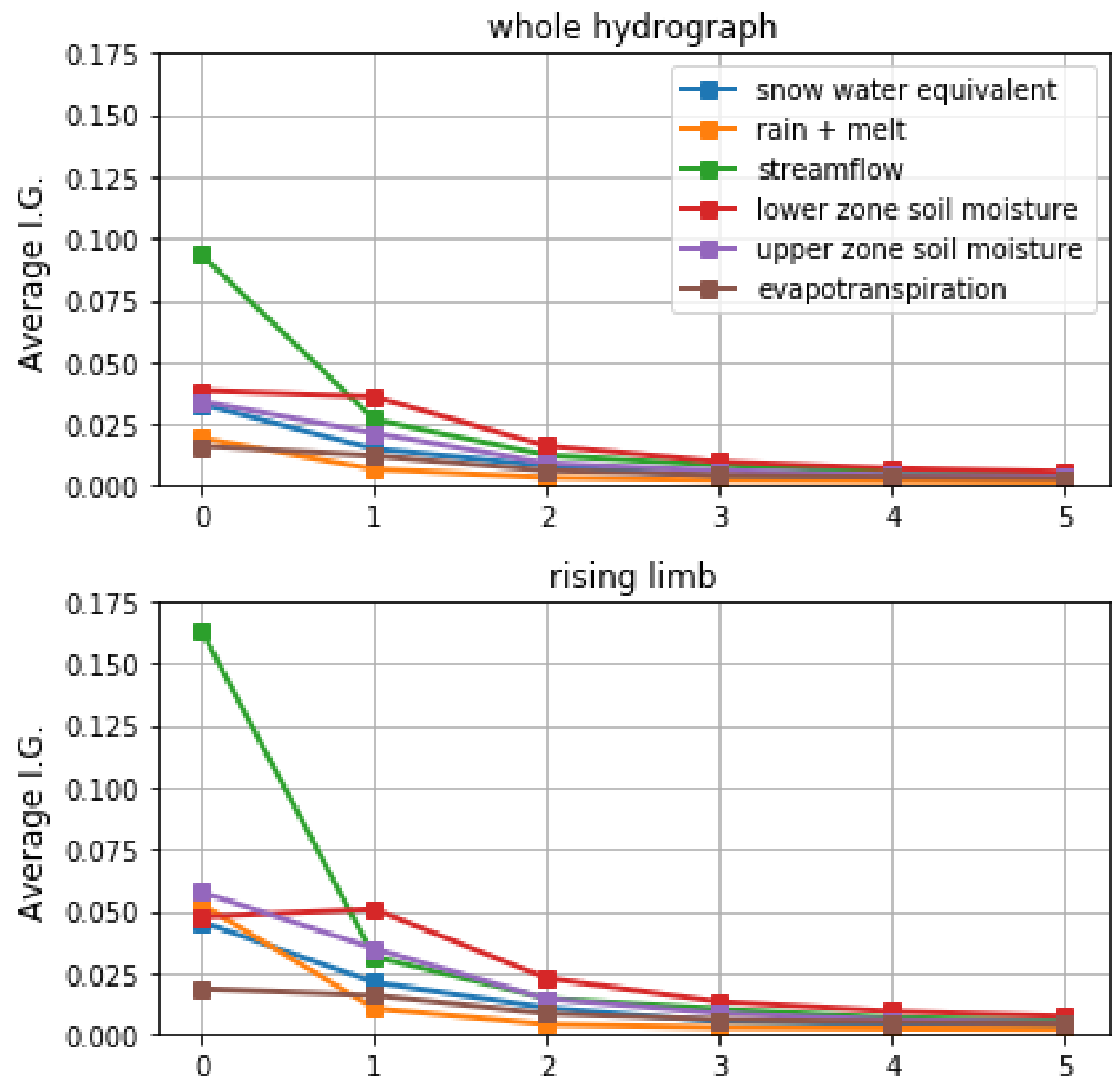

falling limb

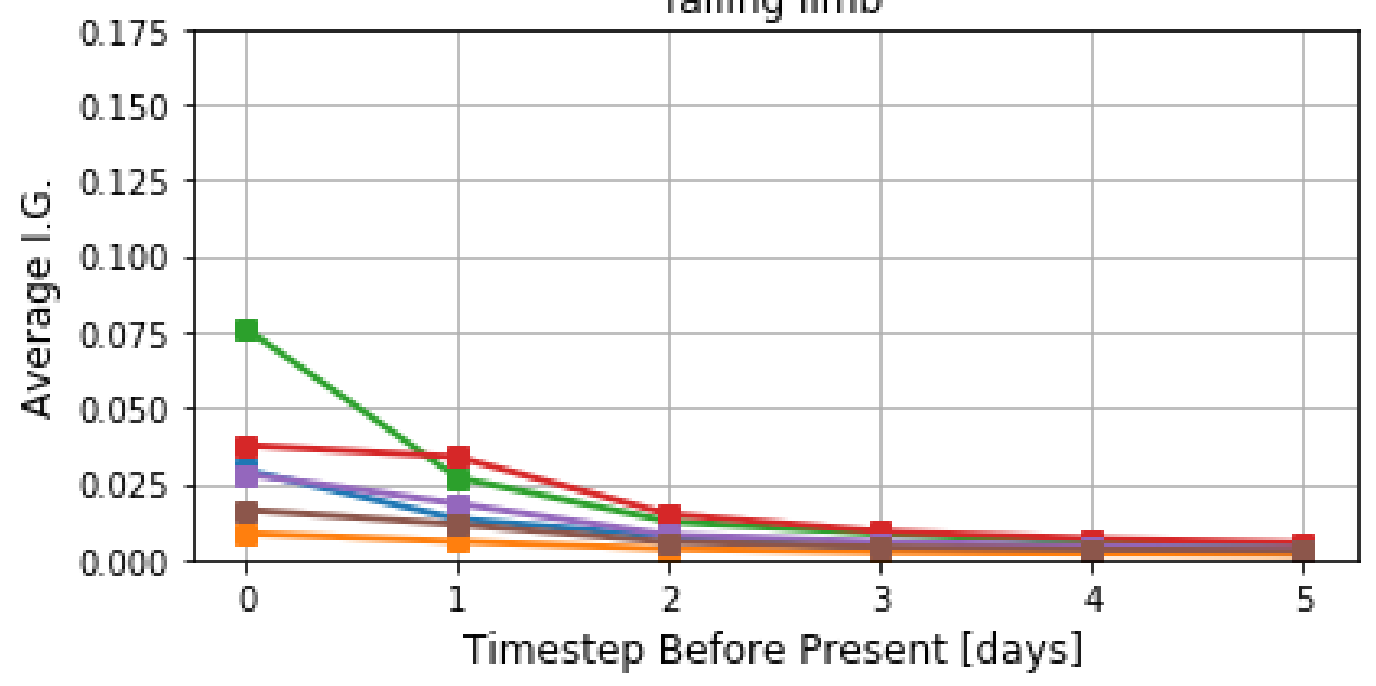

Figure 6. Basin- and time-averaged integrated gradients of only the SAC-SMA related inputs as a function of the first five lookback timesteps. 

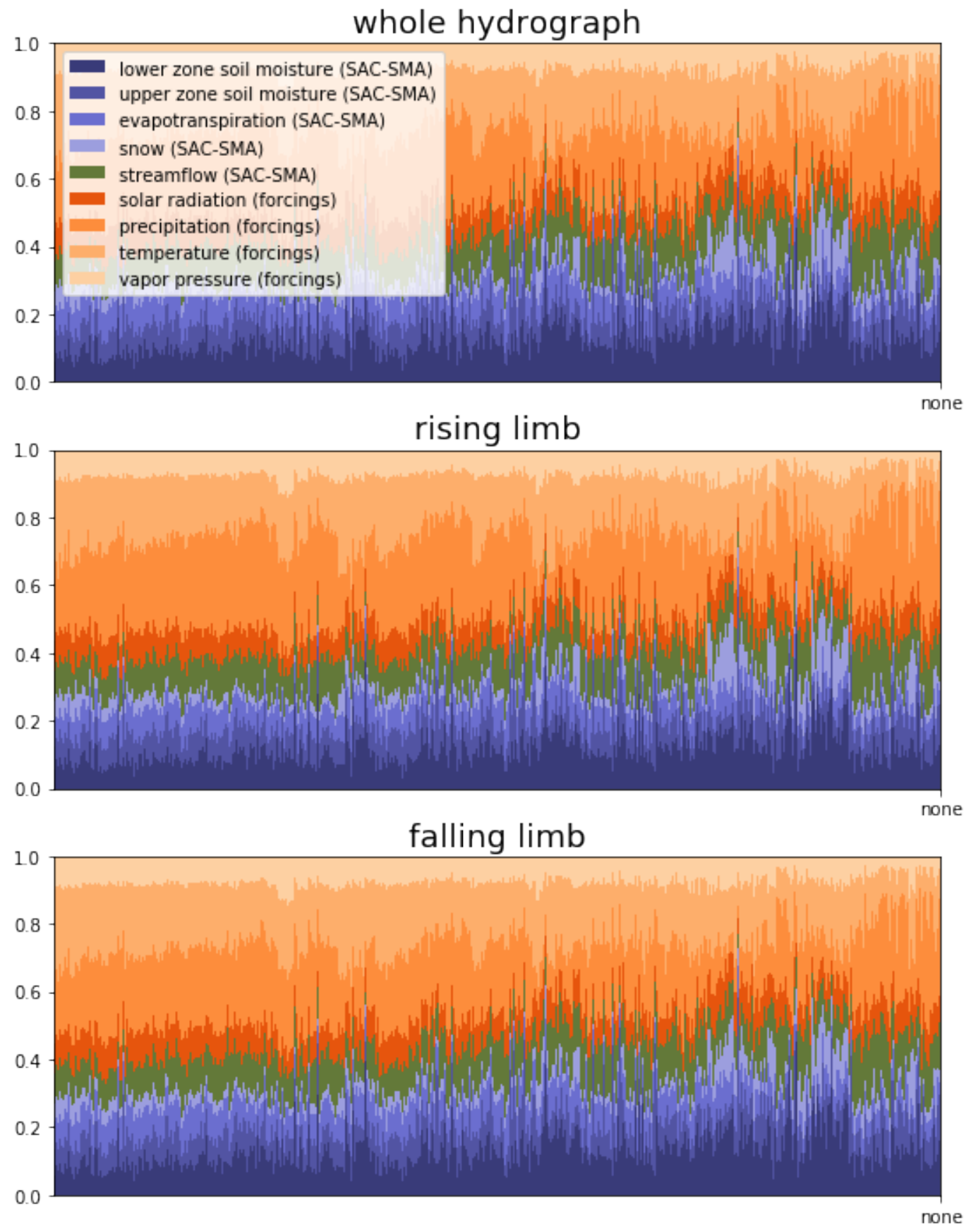

Figure 7. Relative contributions (integrated gradients) of different categories of inputs broken out by basin. The x-axis represents basin ID - this shows diversity between basins, however there is no spatial information in this figure. Orange colors represent meteorological forcings and purple colors represent SAC-SMA related inputs. 

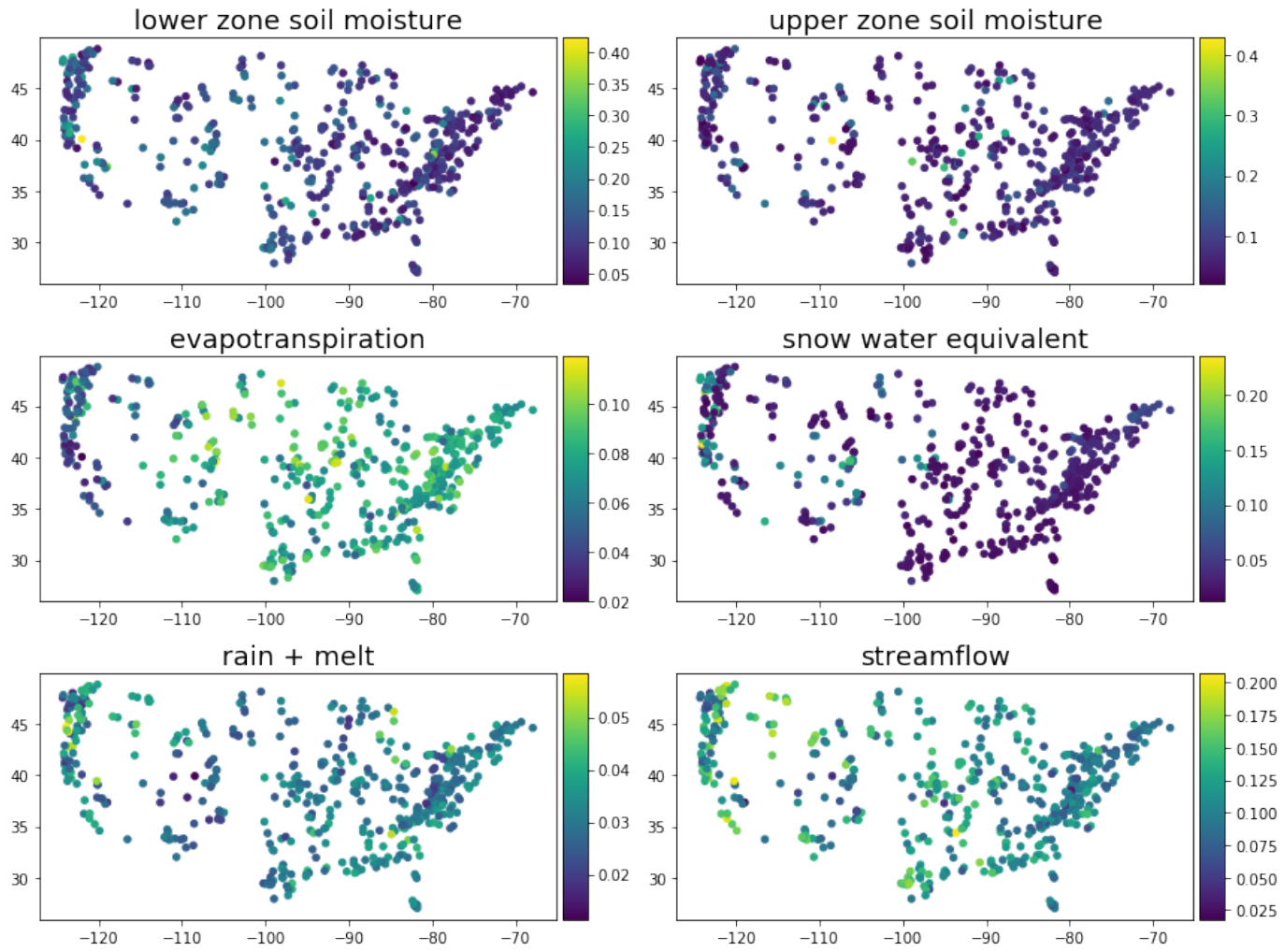

Figure 8. Relative contributions (integrated gradients) of different categories of SAC-SMA related inputs broken out by basin and mapped spatially.

sually in these plots (and indeed, no analysis that we performed found meaningful quantitative spatial patterns), however it is notable that the upper zone soil moisture variables were very important in a few basins. Snow water equivalent was important in basins in the Rocky Mountains and Sierra Nevada's, but not in the Appalachians or northern regions of the US.

\subsection{Predictability}

Figure 9 shows correlations (over 531 basins) between the time-averaged SAC-SMA inputs and the improvement of the LSTM post-processor relative to both control models (LSTM alone and SAC-SMA alone). There is moderately strong positive correlation between catchments with high SWE states (snow water equivalent) and improvements in (1) the rising limb bias of the standalone LSTM and (2) the peak timing of SAC-SMA. Overall, adding SAC-SMA's snow state improved both models. In contrast, there was negative correlation between improvements to the LSTM bias and lower zone soil moisture, indicating that catchments with less deep percolation (and, presumably less baseflow) gained more from in terms of improving long term bias in the LSTM. Finally, improvements over the standalone SAC-SMA were stronger in basins with lower potential evapotranspiration - presumably these are the more water-limited basins.

Figure 10 illustrates the predictability of improvements over SAC-SMA and over the standalone LSTM due to post-processing. Specifically, this figure shows the $r^{2}$ coefficients from $\mathrm{k}$-fold cross validation of random forest regression to predict differences between the two standalone models and the LSTM post-processor. Predictable improvements were mainly to the peak timing error of the standalone SAC-SMA model, which 

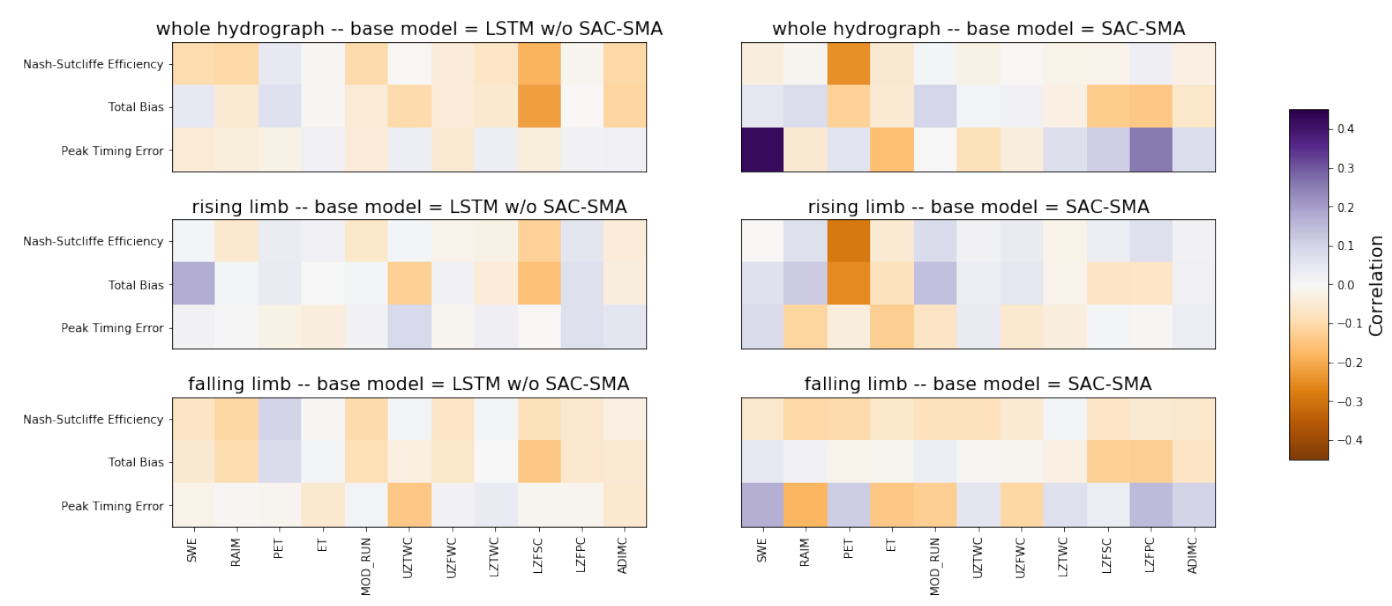

Figure 9. Correlations between the time-averaged SAC-SMA related inputs in each of 531 basins vs. differences in each basin between the LSTM post-processor and both control models (LSTM alone and SAC-SMA alone).

could be predicted using basin-average SAC-SMA inputs, integrated gradients, and to a lesser extent, static catchment attributes. Going back to Figure 9, this is likely due mainly to the fact that there was strong improvement in snow-dominated catchments. Similarly, Figure 11 shows the relative sensitivity to random forest regressions to the basin-averaged SAC-SMA variables. Predictable performance changes were in (1) the peak timing metric of the post-processor relative to the standalone SAC-SMA, and (2) the total bias of the standalone LSTM relative to the post-processor. SWE and lower zone soil moisture were the two most important predictors for these regressions, respectively, again indicating that post-processing helped most (relative to the standalone SAC-SMA) in snow dominated catchments and helped most (relative to the standalone LSTM) in correcting bias in catchments with less deep storage.

\section{Conclusions}

Overall, post-processing by adding SAC-SMA outputs as inputs to an LSTM improved the SAC-SMA model more than it improved the LSTM. SAC-SMA was improved in catchments with more snow, and the standalone LSTM was improved in terms of longterm bias. In general, our analysis indicates that snow processes and baseflow processes are the weakest parts of these models.

Post-processing is arguably the most naive way of combining machine learning with physics-based or conceptual models. This analysis suggests that there is information in even simple conceptual models like SAC-SMA that could be useful for deep learning, however extracting this information in an effective way will require more sophisticated approaches than what we have done here. Given that the LSTM improved in the long-term bias, it is likely that adding explicit conservation constraints to the LSTM architecture might be effective - Nearing et al. (2020) illustrated a general strategy for doing this in deep learning models and with the LSTM in particular.

All of the SAC-SMA implementations here were calibrated to long data records in each basin. This limits applicability of this type of strategy to gauged basins. Future work should explore both post-processing and also more sophisticated methods for model fusion with true process-based hydrology models that may not be as reliant on parameter estimation. 


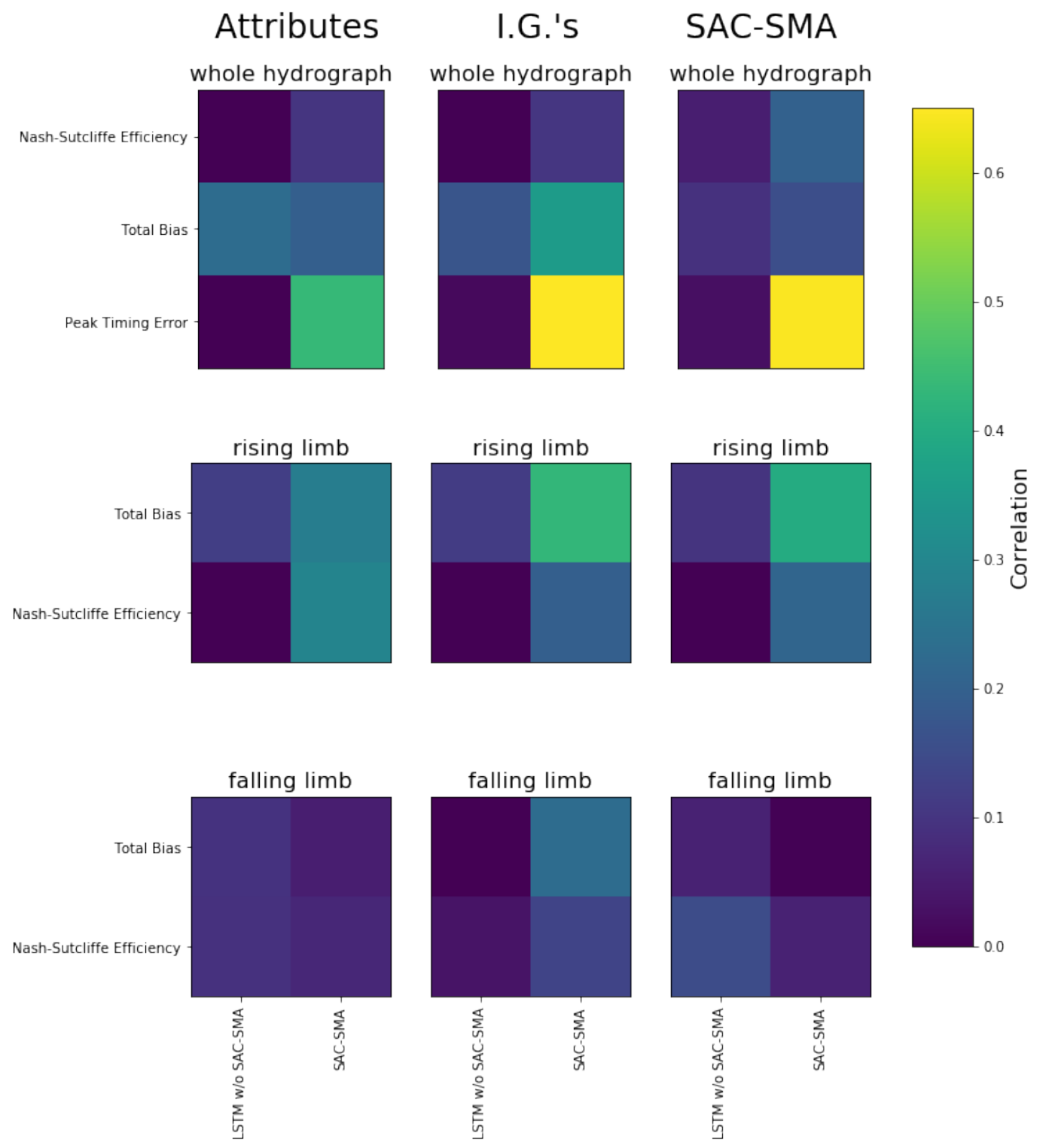

Figure 10. $r^{2}$ statistics from k-fold cross validation of random forest regression to predict differences in performance metrics between standalone models and the LSTM post-processor over different parts of the hydrograph. 

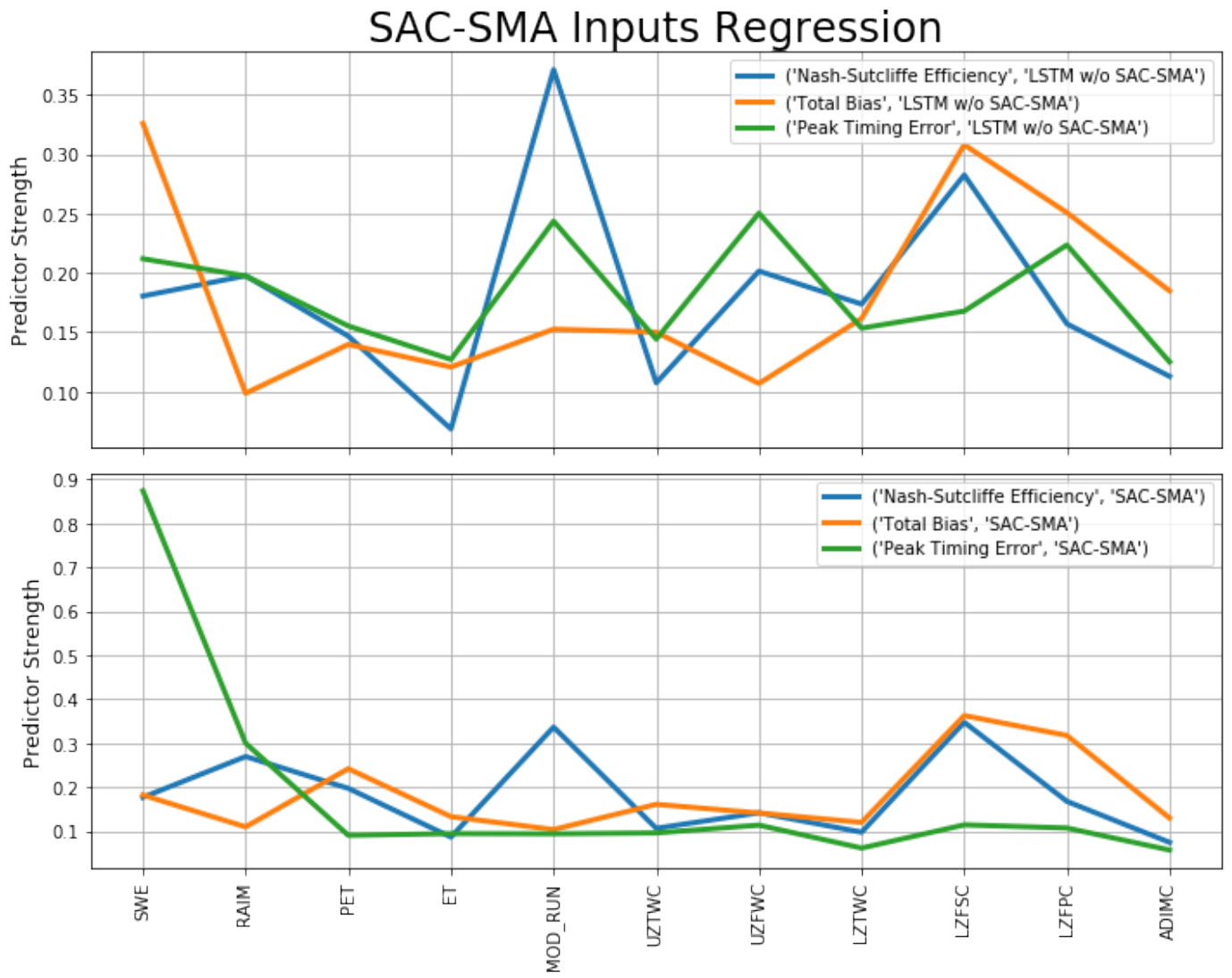

Figure 11. Relative contribution (sensitivity) of different SAC-SMA variables as inputs to random forest regressions used to predict differences in performance scores with vs. without post-processing. Predictable performance changes were in (1) the peak timing metric of the postprocessor relative to the standalone SAC-SMA, and (2) the total bias of the standalone LSTM relative to the post-processor. 
Data Availability Statement: Data for this project came from the CAMELS repository hosted by the US National Center for Atmospheric Research at https://ral .ucar.edu/solutions/products/camels

Code Availability Statement: All code necessary to reproduce results is available at https://github.com/kratzert/lstm_based_hydrology/tree/sacsma_postprocessing

\section{Acknowledgments}

Authors from the Johannes Kepler University were partially supported by a Google faculty research award. Jonathan Frame from the University of Alabama was partially supported by the NASA Terrestrial Hydrology Program.

\section{References}

Addor, N., Nearing, G., Prieto, C., Newman, A., Le Vine, N., \& Clark, M. P. (2018). A ranking of hydrological signatures based on their predictability in space. Water Resources Research, 54(11), 8792-8812.

Addor, N., Newman, A. J., Mizukami, N., \& Clark, M. P. (2017). The camels data set: catchment attributes and meteorology for large-sample studies. Hydrology and Earth System Sciences (HESS), $21(10), 5293-5313$.

Burnash, R. J., Ferral, R. L., \& McGuire, R. A. (1973). A generalized streamflow simulation system: Conceptual modeling for digital computers. US Department of Commerce, National Weather Service, and State of California ....

Daw, A., Thomas, R. Q., Carey, C. C., Read, J. S., Appling, A. P., \& Karpatne, A. (2020). Physics-guided architecture (pga) of neural networks for quantifying uncertainty in lake temperature modeling. In Proceedings of the 2020 siam international conference on data mining (pp. 532-540).

Duan, Q., Gupta, V. K., \& Sorooshian, S. (1993). Shuffled complex evolution approach for effective and efficient global minimization. Journal of optimization theory and applications, $76(3), 501-521$.

Hochreiter, S., \& Schmidhuber, J. (1997). Long short-term memory. Neural Computation, $9(8), 1735-1780$. Retrieved from https://doi.org/10.1162/neco .1997.9.8.1735 doi: 10.1162/neco.1997.9.8.1735

Kratzert, F., Herrnegger, M., Klotz, D., Hochreiter, S., \& Klambauer, G. (2019). Neuralhydrology-interpreting lstms in hydrology. In Explainable ai: Interpreting, explaining and visualizing deep learning (pp. 347-362). Springer.

Kratzert, F., Klotz, D., Herrnegger, M., Sampson, A. K., Hochreiter, S., \& Nearing, G. (2019). Toward improved predictions in ungauged basins: Exploiting the power of machine learning. Water Resources Research.

Kratzert, F., Klotz, D., Hochreiter, S., \& Nearing, G. (2020). A note on leveraging synergy in multiple meteorological datasets with deep learning for rainfallrunoff modeling. Hydrology and Earth System Sciences Discussions.

Kratzert, F., Klotz, D., Shalev, G., Klambauer, G., Hochreiter, S., \& Nearing, G. (2019). Towards learning universal, regional, and local hydrological behaviors via machine learning applied to large-sample datasets. Hydrology and Earth System Sciences, 23(12), 5089-5110.

McMillan, H., Westerberg, I., \& Branger, F. (2017). Five guidelines for selecting hydrological signatures.

Nearing, G., Kratzert, F., Sampson, A. K., Pelissier, C., Klotz, D., Frame, J., \& Gupta, H. (2020). What role does hydrological science play in the age of machine learning?

Newman, A., Clark, M., Sampson, K., Wood, A., Hay, L., Bock, A., ... others (2015). Development of a large-sample watershed-scale hydrometeorological data set for the contiguous usa: data set characteristics and assessment of regional variability in hydrologic model performance.

Hydrology and Earth 
System Sciences, 19(1), 209-223.

Pelissier, C., Frame, J., \& Nearing, G. (2020). Combining parametric land surface models with machine learning. arXiv preprint arXiv:2002.06141.

Roe, J., Dietz, C., Restrepo, P., Halquist, J., Hartman, R., Horwood, R., ... Welles, E. (2010). Noaa's community hydrologic prediction system. In Proceedings from the 4 th federal interagency hydrologic modeling conference.

Sundararajan, M., Taly, A., \& Yan, Q. (2017). Axiomatic attribution for deep networks. In Proceedings of the 34th international conference on machine learning-volume 70 (pp. 3319-3328). 